ApplicationForum

\title{
Dextran Sodium Sulfate for colitis animal model research MPBiomedicals, CA, USA
}

Inflammatory bowel disease (IBD) is a chronic, remitting and relapsing inflammatory disorder encompassing ulcerative colitis (UC) and Crohn's disease.

Conversely, Crohn's disease can affect any part of gastrointestinal tract, but the most commonly affected regions include the terminal ileum or the perianal region. Clinically, IBD is characterized by severe diarrhea, bleeding, abdominal pain, fluid and electrolyte loss, reflecting the underlying inflammatory process. Crohn's disease is characterized by activation of immune response of a Th1-phenotype. On the other hand, ulcerative colitis portrays Th-2 driven immune response.

In past decades, dozens of different animal models of IBD have been developed. These models can be broadly divided into spontaneous colitis models, inducible colitis models, genetically modified models, and adoptive transfer models. Although these models do not represent the complexity of human disease, they are valuable and indispensable tools that provide a wide range of options for investigating involvement of various factors into the pathogenesis of IBD and evaluate different therapeutic options. Chemically induced murine models of intestinal inflammation are one of the most commonly used models because they are simple to induce, and the onset, duration, and severity of inflammation are immediate and controllable.

DSS causes erosions with complete loss of surface epithelium because of its direct toxic effect on epithelial cells. It causes deformity in the epithelial integrity, thereby increasing the colonic mucosal permeability allowing permeation of large molecules, such as DSS, with molecular weight up to 50,000 Da.

The DSS-induced colitis model has some advantages when compared to other animal models of colitis. For example, an acute, chronic, or relapsing model can be produced easily by changing the concentration of administration of DSS (and cycle in rats and other strains of mice). Moreover, dysplasia that resembles the clinical course of human UC occurs frequently in the chronic phase of DSS-induced colitis. The DSS-induced model for studying colitis-associated carcinogenesis has recently been reviewed by others. Furthermore, studies that validated the DSS model by using different therapeutic agents for human IBD show that DSS-induced colitis can be used as a relevant model for the translation of mice data to human disease.

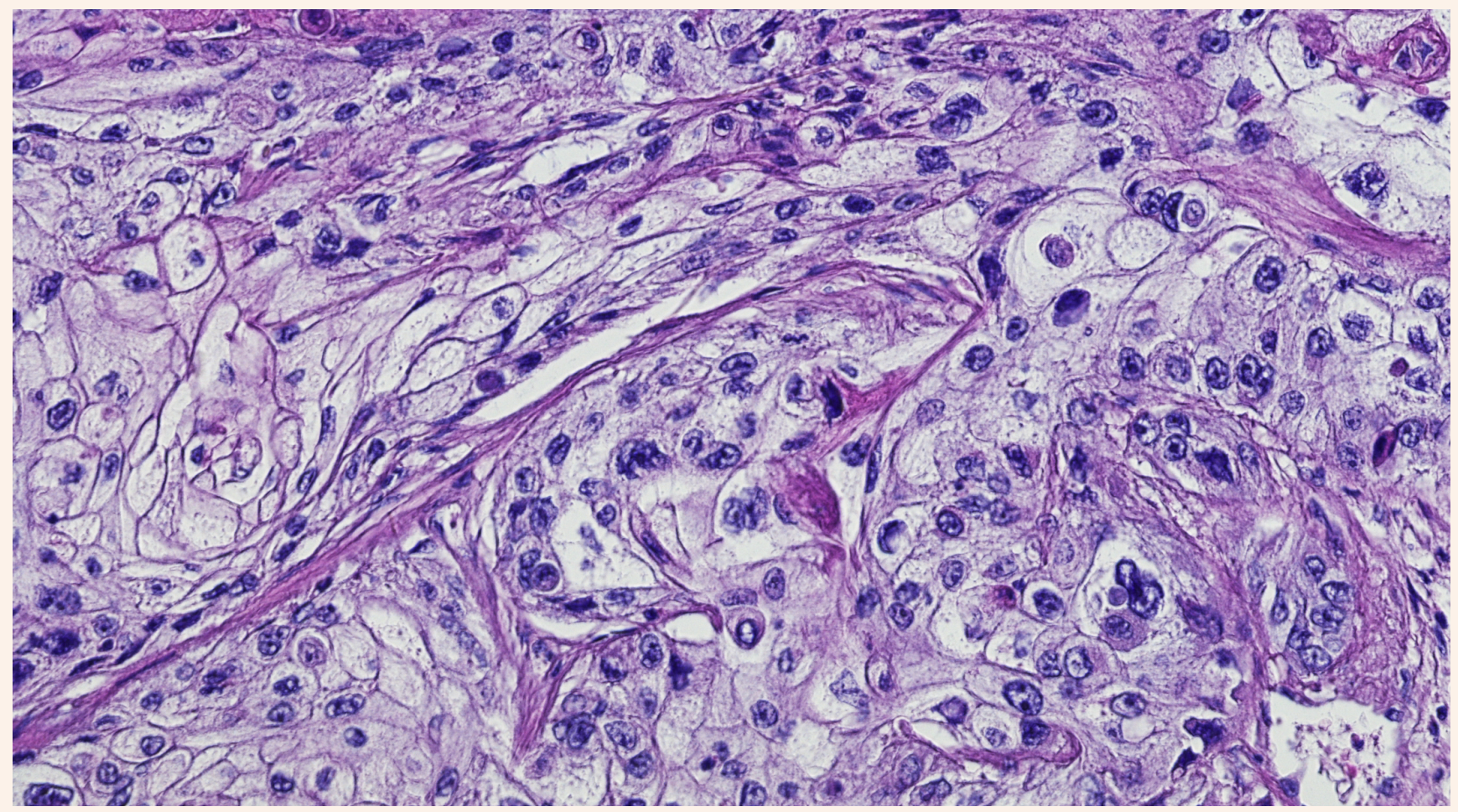

BioTechniques 64:185-186 (April 2018) doi 10.4155/btn-2017-0128 


\begin{tabular}{|c|c|c|c|}
\hline Mice strains & Dose & Days & Publication \\
\hline C57BL/6 & $2.5 \%$ & 8 & $\begin{array}{l}\text { Dietary fish oil and curcumin combine to modulate colonic } \\
\text { cytokinetics and gene expression in dextran sulfate sodium } \\
\text { treated mice Q.Jia, et al., Br. J. Nutr., 2011;106(4),519-9. }\end{array}$ \\
\hline Wild-type C57BL/6J(m) & $3 \%$ & 6 & $\begin{array}{l}\text { Nanoparticle-based therapeutic delivery of prohibiting to the } \\
\text { colonic epithelial cells ameliorates acute murine colitis A.L. } \\
\text { Thiess, et al., Inflamm. Bowel Dis., 2011; } 17(5), 1163-76 .\end{array}$ \\
\hline C57BL/6 AhR null, WT & $3.5 \%$ & 7 & $\begin{array}{l}\text { Role of xenobiotic receptor in inflammatory bowel disease, R. } \\
\text { Arsenescu, et al, Inflamm. Bowel Dis., 2011; 17(5), 1149-2. }\end{array}$ \\
\hline C57BL/6 & $5 \%$ & 7 & $\begin{array}{l}\text { Microbial ecology of the murine gut associated with the } \\
\text { development of dextran sulfate sodium induced colitis N.A. } \\
\text { Nagalingham, et al., Inflamm, Bowel Disease, 2011; 7(4), 917-26. }\end{array}$ \\
\hline Balb/c & $1 \%$ & 10 & $\begin{array}{l}\text { Salmonella-mediated gene therapy in experimental colitis in } \\
\text { mice, R.Pal_y, et al, Ex.Biol.Med., 2011; 236(2), 177-83. }\end{array}$ \\
\hline Balb/c & $3 \%$ & 5 & $\begin{array}{l}\text { GCMS-based metabolomic study in mice with colitis induced by } \\
\text { dextran sulfate sodium. Y.Shiomi, et al., Inflamm. Bowel } \\
\text { Dis. } 2011 ; 17(11), 2261-74 .\end{array}$ \\
\hline BALB/c & $1-5 \%$ & 10 & $\begin{array}{l}\text { Anti-inflammatory effects of Lactobacillus casei BL23 producing } \\
\text { or not a manganese-dependent catalase on DSS-induced colitis } \\
\text { in mice, T.Rochat, et al., Microb. CellFact., } 2007 ; 20(6), 22 .\end{array}$ \\
\hline WT; CCR9(-/-); CCL25 (-/-) & $2 \%$ & 7 & $\begin{array}{l}\text { CCL25/CCR9 interactions regulate large intestinal inflammation } \\
\text { in a murine model of acute colitis, M.A. Wurbel, et al., PLoS } \\
\text { One, 2011; 6(1), e16442. }\end{array}$ \\
\hline Wild-type; DPIV -/- & $2 \%$ & 6 & $\begin{array}{l}\text { Biochemical and histological changes in the small intestine of } \\
\text { mice with dextran sulfate sodium induced colitis, R.Yazbeck, et } \\
\text { al., J.Cell Physiol., 2011; 226(12), 319-24. }\end{array}$ \\
\hline C57BL//6 & $5 \%$ & 7 & $\begin{array}{l}\text { N.M. Kulkarni, Embelin ameliorates dextran sulfate sodium } \\
\text { induced colitis 14. 26. G.K.Kumar, R.Dhamotharan. in mice, Int. } \\
\text { Immunopharmacol., } 2011 \text {. E }\end{array}$ \\
\hline
\end{tabular}

\begin{tabular}{|c|c|c|c|}
\hline Rat Strains & Dose & Days & Publication \\
\hline Sprague-Dawley & $5 \%$ & 9 & $\begin{array}{l}\text { Lactobacillus reuteri maintains a functional mucosal barrier during DSS treatment } \\
\text { despite mucus layer dysfunction J.Dicksved, et al., PLoS One, 2012; 7(9):e46399. }\end{array}$ \\
\hline Sprague-Dawley & $5 \%$ & 6 & $\begin{array}{l}\text { Non-peptidyl low molecular weight radical scavenger IAC attenuates DSS-induced } \\
\text { colitis in rats.V.Vasina et al., World J.Gastroenterol., 2010; 16(29), 3642-50. }\end{array}$ \\
\hline Sprague-Dawley & $5 \%$ & 7 & $\begin{array}{l}\text { Differential immune and genetic responses in rat models of Chrohn's colitis and } \\
\text { ulcerative colitis X.Z.Shi, et al., Am.J.Physiol.Gastrointest.Liver Physiol., 2011; } \\
300(1) \text {, G41-51. }\end{array}$ \\
\hline Wistar & $5 \%$ & 10 & $\begin{array}{l}\text { Bovine glucomacropeptide has intestinal anti-inflammatory effects in rats with } \\
\text { dextran-sulfate induced colitis R.Lopez-Posadeas, et al., J.Nutr., 2010; 14O(11), } \\
2014-2019 .\end{array}$ \\
\hline Wistar & $2-4 \%$ & 7 & $\begin{array}{l}\text { The relationship between the concentration of dextran sodium sulfate and the } \\
\text { degree of induced experimental colitis in weanling rats T.Shimizu, et al., } \\
\text { J.Pediatric Gastro. Nutrition, } 2003 ; 37,481-486 \text {. }\end{array}$ \\
\hline
\end{tabular}

\begin{tabular}{|cccl|}
\hline Other & Dose & Days & \multicolumn{1}{c|}{ Publication } \\
\hline Hamster & $2.5 \%$ & 6 & $\begin{array}{l}\text { Dextran sulphate sodium induces acute colitis and alters hepatic function in } \\
\text { hamsters A.Karlsson, et al., Int. Immunopharmacol., 2008; 8(1), 20-27. }\end{array}$ \\
\hline Hamster & $1 \%$ & $\begin{array}{l}\text { Lactobacillus reuteri maintains a functional mucosal barrier during DSS } \\
\text { treatment despite mucus layer dysfunction J.Dicksved, et al., PLoS One, 2012; } \\
7(9): \text { e46399. }\end{array}$ \\
\hline Guinea Pig & $3 \%$ & 4 & $\begin{array}{l}\text { Morphological analysis of acute ulcerous colitis experimentally induced by } \\
\text { dextran sulfate sodium in the guinea pig T.Iwanaga, O et al., J. Gastroenterol., } \\
1994 ; 430-438 .\end{array}$ \\
\hline $\begin{array}{c}\text { Pig } \\
\text { (Yorkshire) }\end{array}$ & $\begin{array}{c}1.25 \mathrm{~g} / \mathrm{kg} \\
\mathrm{BW}\end{array}$ & 5 & $\begin{array}{l}\text { Soy-derived di-and tripeptides alleviate colon and ileum inflammation in pigs } \\
\text { with dextran sodium sulfate-induced colitis D.Young, et al., J.Nutr., } \\
2012 ; 142(2), 363-8 .\end{array}$ \\
\hline
\end{tabular}

